\title{
Adenoviral vector-mediated expression of a gene encoding secreted, EpCAM-targeted carboxylesterase-2 sensitises colon cancer spheroids to CPT- I I
}

\section{Oosterhoff",', RM Overmeer', M de Graaf', IH van der Meulen', G Giaccone', VW van Beusechem', HJ Haisma ${ }^{2}$, HM Pinedo' and WR Gerritsen'}

'Department of Medical Oncology, Division of Gene Therapy, VU University Medical Center, PO Box 7057, 1007 MB Amsterdam, The Netherlands;

${ }^{2}$ Department of Therapeutic Gene Modulation, University Center for Pharmacy, PO Box 196, 9700 AD Groningen, The Netherlands

\begin{abstract}
CPT-II (irinotecan or 7-ethyl-IO[4-(I-piperidino)-I-piperidino] carbonyloxycamptothecin) is an anticancer agent in use for the treatment of colon cancer. In order to be fully active, CPT-I I needs to be converted into SN-38 (7-ethyl- I0-hydroxycamptothecin) by the enzyme carboxylesterase (CE). In humans, only a minority of CPT- I I is converted to SN-38. To increase the antitumour effect of CPT-II by gene-directed enzyme prodrug therapy, we constructed a replication-deficient adenoviral vector Ad.C28-sCE2 containing a fusion gene encoding a secreted form of human liver CE2 targeted to the surface antigen epithelial cell adhesion molecule (EpCAM) that is highly expressed on most colon carcinoma cells. By targeting CE2 to EpCAM, the enzyme should accumulate specifically in tumours and leakage into the circulation should be minimised. Ad.C28-sCE2-transduced colon carcinoma cells expressed and secreted active CE that bound specifically to EpCAM-expressing cells. In sections of three-dimensional colon carcinoma spheroids transduced with Ad.C28-sCE2, it was shown that C28-sCE2 was capable of binding untransduced cells. Most importantly, treatment of these spheroids with nontoxic concentrations of CPT-I I resulted in growth inhibition comparable to treatment with SN-38. Therefore, Ad.C28-sCE2 holds promise in gene therapy approaches for the treatment of colon carcinoma. British Journal of Cancer (2005) 92, 882-887. doi:I0.1038/sj.bjc.6602362 www.bjcancer.com
\end{abstract}

Published online I March 2005

(c) 2005 Cancer Research UK

Keywords: adenovirus; carboxylesterase; CPT-II; EpCAM; spheroid

Conventional chemotherapy is not specific for tumour cells and therefore its administration is limited by side effects. These side effects might potentially be overcome by targeting chemotherapy specifically to tumour cells by gene-directed enzyme prodrug therapy (GDEPT). In GDEPT, a gene encoding a prodrugconverting enzyme is delivered to the tumour by, for example, an adenoviral vector. If the prodrug is administered it will be specifically converted to the active drug at the site of the tumour. This should increase the efficacy and decrease the side effects of chemotherapy. CPT-11 (irinotecan or 7-ethyl-10[4-(1-piperidino)1-piperidino] carbonyloxycamptothecin) is an anticancer agent that is approved for first-line treatment of metastatic colon cancer. In order to be fully active, CPT-11 needs to be activated into the active compound SN-38 (7-ethyl-10-hydroxycamptothecin) by carboxylesterase (CE) enzymes (Tsuji et al, 1991; Satoh et al, 1994). Although SN-38 is detected in the plasma of cancer patients only minutes after administration of CPT-11 (Gupta et al, 1997), $90-95 \%$ of the prodrug is not converted to SN-38 (Rivory et al, 1997). A way to improve the antitumour effect of CPT-11 may be to use CPT-11 and CE in a GDEPT approach. Adenoviral-mediated

*Correspondence: Dr D Oosterhoff; E-mail: d.oosterhoff@vumc.n Received 20 July 2004; revised 15 November 2004; accepted 25 November 2004; published online I March 2005 expression of rabbit CE showed to sensitise efficiently a panel of tumour cell lines to CPT-11 (Wierdl et al, 2001). A human enzyme, however, has the advantage over a non-human enzyme that it will not lead to an immune response against the enzyme and subsequent enzyme inactivation. Kojima et al (1998) described the construction of a replication-deficient adenoviral vector containing the cDNA encoding human liver CE isoform 1 (CE1) (Kojima et al, 1998). Cell lines transduced with this virus and treated with CPT-11, however, showed only minimal antitumour effects. The liver CE isoform 2 (CE2) has a higher affinity and a higher conversion velocity of CPT-11 compared to CE1 (Humerickhouse et al, 2000). Therefore, we envisaged that human liver CE2 would be the best candidate to employ in a GDEPT approach to treat human colon cancer. Given the fact that current gene transfer technologies do not allow transduction of all tumour cells, a bystander effect is warranted to achieve effective kill of untransduced tumour cells. To improve the bystander effect of adenoviral vector-mediated GDEPT approaches, secreted and surface-tethered prodrug-converting enzymes have been investigated (Spooner et al, 2000; Weyel et al, 2000; Heine et al, 2001; Oosterhoff et al, 2003). We envisioned that a targeted, secreted form of CE2, consisting of the secreted form of CE2 (sCE2) fused to a tumour-specific $\mathrm{scFv}$ antibody would provide an enlarged bystander effect and would furthermore theoretically prevent leakage of the protein into the circulation, thereby reducing 
systemic side effects. Previously, we constructed a fusion protein in which sCE2 was fused to the human scFv antibody C28, which is directed to the tumour antigen Epithelial Cell Adhesion Molecule (EpCAM) (Oosterhoff et al, 2002). This fusion protein has potential utility for GDEPT of colon cancer, because EpCAM is highly overexpressed in colon cancer cells including distant metastases (Litvinov et al, 1994). Here, we describe the construction of a replication-deficient adenoviral vector containing the cDNA encoding the fully human fusion protein C28-sCE2. In a three-dimensional tumour spheroid model in vitro, we could demonstrate that the secreted fusion protein bound nontransduced cells and caused efficient killing of colon cancer cells in the presence of CPT- 11 .

\section{MATERIALS AND METHODS}

\section{Cells and culture conditions}

The colon cancer cell lines SW1398 and Colo205 and the ovarian cancer cell line A2780 (all cell lines were kindly provided by Dr E Boven, VUMC, Amsterdam, The Netherlands) were maintained in Dulbecco's modified Eagle's medium (DMEM) supplemented with $10 \%$ foetal calf serum (FCS), $50 \mathrm{IU} \mathrm{ml}^{-1}$ penicillin and $50 \mu \mathrm{g} \mathrm{ml}^{-1}$ streptomycin (Invitrogen, Breda, The Netherlands), at $37^{\circ} \mathrm{C}$ in a $5 \% \mathrm{CO}_{2}$ humidified atmosphere. The 293 cell line (ATCC, Manassas, VA, USA) was maintained in DMEM supplemented with $10 \% \mathrm{FCS}, 50 \mathrm{IU} \mathrm{ml}^{-1}$ penicillin, $50 \mu \mathrm{g} \mathrm{ml}^{-1}$ streptomycin and 2 mM L-glutamine (Invitrogen).

\section{Formation of colon cancer spheroids}

In all 96-well plates (Greiner, Alphen aan den Rijn, The Netherlands) were coated with $2 \%$ agarose (Roche, Almere, The Netherlands) in PBS. Colon cancer SW1398 or Colo205 cells were plated $\left(1 \times 10^{4}\right.$ cells well $\left.^{-1}\right)$ and rotated overnight at 140 r.p.m. in a Heidolph Unimax incubator. By plating equal amounts of cells in each well and rotating them overnight, spheroids of similar sizes are formed. This allows direct comparison of different treatment modalities. After rotation, the formed spheroids were grown in a $5 \% \mathrm{CO}_{2}$ humidified atmosphere at $37^{\circ} \mathrm{C}$ for 3 days before use in transduction experiments.

\section{Construction of Ad.C28-sCE2}

The adenoviral vector Ad.C28-sCE2 was constructed using the AdEasy System (He et al, 1998). The plasmid pSTCF-C28-sCE2, containing the secreted, EpCAM-targeted CE2 (C28-sCE2) open reading frame (Oosterhoff et al, 2002) with a myc-6His tag at the C-terminus, was digested with PmeI and NheI, and the C28-sCE2 open reading frame was ligated into the $X b a \mathrm{I}-$ and EcoRVlinearised transfer vector pAdTrack-CMV. This construct contains a gene encoding green fluorescent protein (GFP) under the CMV promoter. Subsequently, the plasmid was digested with $P m e I$ and cotransformed into Escherichia coli BJ5183 cells with adenoviral backbone plasmid pAdEasy-1 to construct pAdEasy-C28-sCE2. After linearisation of this recombinant vector with PacI, the plasmid was transfected into the 293 adenovirus packaging cell line. Virus was further propagated in 293 cells according to standard techniques. For all experiments, AdGFP (van Beusechem et al, 2000) was taken along as a negative control.

\section{Western blot analysis}

Equivalent amounts of supernatant or cellular lysate from SW1398 cells transduced with Ad.C28-sCE2 were dissolved in sample buffer (Laemmli, 1970) with 2-mercaptoethanol and heated to $95^{\circ} \mathrm{C}$ for $5 \mathrm{~min}$. Samples were electrophoresed through a denaturing $10 \%$ sodium dodecyl sulphate-polyacrylamide gel and protein bands were electroblotted onto a PVDF protein membrane (BioRad, Veenendaal, The Netherlands). Proteins were detected using antimyc antibody 9E10 (Chan et al, 1987) and HRP-conjugated rabbit anti-mouse IgG (DakoCytomation, Heverlee, Belgium). Films were developed with enhanced chemoluminescence (Lumilight Plus, Roche, The Netherlands).

\section{Esterase activity assay}

To evaluate the esterase activity of proteins expressed by SW1398 cells transduced with Ad.C28-sCE2, cellular lysates or supernatants were incubated with $200 \mu \mathrm{l} 100 \mathrm{~mm}$ Tris- $\mathrm{HCl}(\mathrm{pH}=8.0)$ containing $1 \mathrm{~mm} p$-nitrophenyl-acetate (pNpAc) (Sigma Aldrich, Zwijndrecht, The Netherlands), a substrate for CE. Conversion to $\mathrm{pNp}$ at room temperature was measured during $10 \mathrm{~min}$ using an ELISA plate reader (BioRad) at a wavelength of $415 \mathrm{~nm}$.

\section{Immunohistochemistry}

Spheroids were harvested at different time points after transduction (day 1, 4 or 5) in TissueTek (Sakura Finetek, Zoeterwoude, The Netherlands) and cryostat sections of $7-10 \mu \mathrm{m}$ were made and stored at $-80^{\circ} \mathrm{C}$. After drying, sections were fixed with $4 \%$ formaldehyde in PBS for $30 \mathrm{~min}$, washed with PBS and treated with $0.2 \%$ Triton $\mathrm{X}-100$ in PBS. After washing, the sections were incubated for $1 \mathrm{~h}$ with the anti-myc antibody 9E10. As a positive control, anti-EpCAM antibody 323A3 (kindly provided by Centocor, Leiden, The Netherlands) was taken along, and as negative controls, PBS/0.1\% BSA and anti-glucuronidase (Haisma et al, 1995) were used. After incubation, sections were washed with PBS and incubated with rabbit-anti-mouse-HRP or goat-antirabbit-HRP (1:100 in PBS/0.1\% BSA, both from DakoCytomation). After incubation for $1 \mathrm{~h}$, sections were washed with PBS and stained with AEC (DakoCytomation) and sections were counterstained with haematoxylin.

\section{In vitro cytotoxicity assays}

At 3 days after the formation of colon cancer spheroids, the spheroids were transduced with $1 \times 10^{7}$ plaque-forming units (PFU) Ad.C28-sCE2 in $100 \mu$ l culture medium. Control spheroids were transduced with AdGFP or cultured in medium. After 7 days, $100 \mu \mathrm{l}$ culture medium was added containing a range of CPT-11 (Aventis, Strasbourg, France). After a further 7 days, cell viability was determined by WST-1 (Roche Diagnostics) conversion at $37^{\circ} \mathrm{C}$. Data are expressed as percentages compared to untransduced, untreated control spheroids.

\section{RESULTS}

\section{Construction and characterisation of Ad.C28-sCE2}

The open reading frame of C28-sCE2 with C-terminal mycHis-tag was inserted in place of the $\mathrm{E} 1$ region of an adenovirus vector next to a GFP expression cassette to create Ad.C28-sCE2 (Figure 1A).

SW1398 colon cancer cells were transduced with Ad.C28-sCE2 or control virus AdGFP at an MOI of 100 and after 6 days expression of sCE2 in supernatant and cellular lysate was analysed by Western blotting. Figure $1 \mathrm{~B}$ shows that the majority of the $110 \mathrm{kDa}$ C28-sCE2 protein was detected in the supernatant of Ad.C28-sCE2-transduced cells, confirming efficient secretion. Enzyme activity of C28-sCE2 was demonstrated by an esterase enzyme activity assay (Figure 1C). Binding of C28-sCE2 to EpCAM-expressing cells was shown by immunohistochemistry (Figure 1D). The EpCAM-positive cell line Colo205 and the EpCAM-negative ovarian cancer cell line A2780 were incubated 


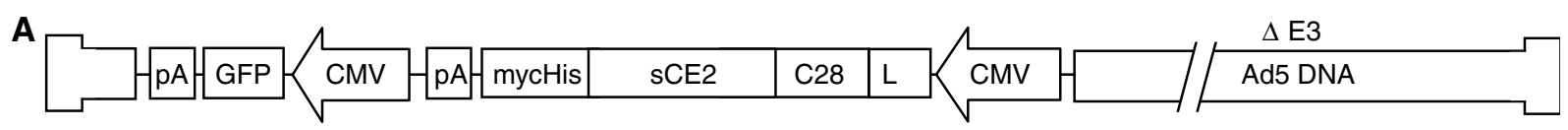

B
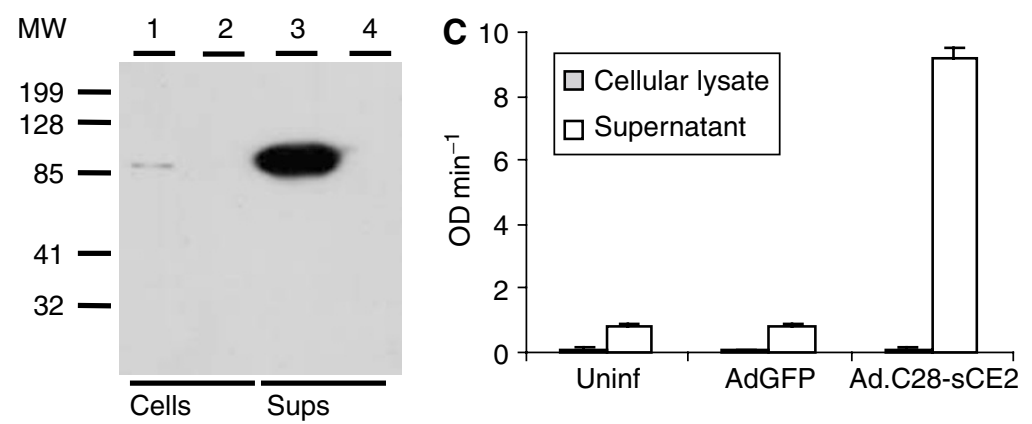

D
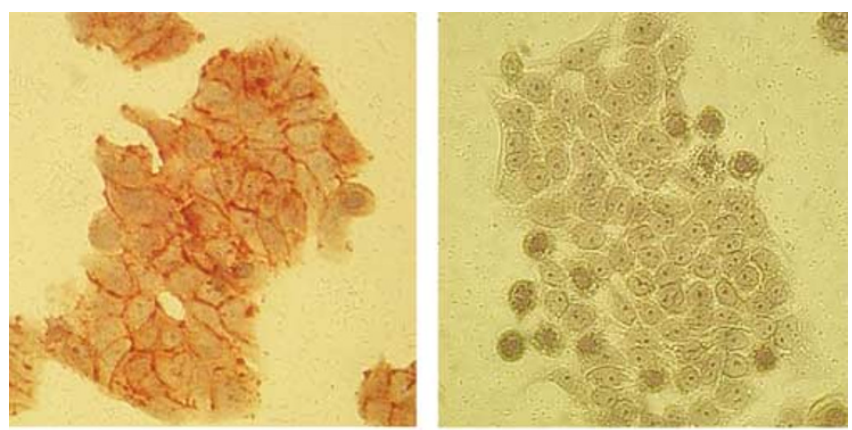

Colo205

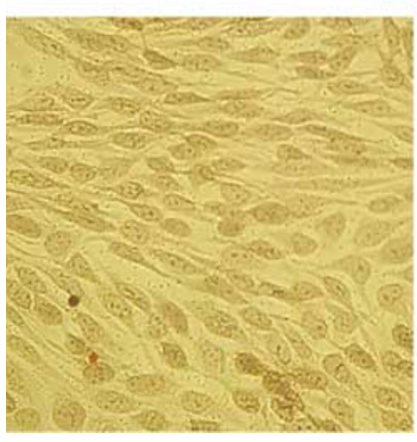

Ad.C28-sCE2

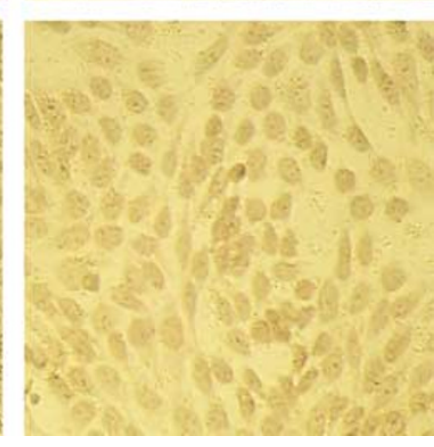

AdGFP

Figure I Schematic structure of the replication-deficient adenovirus Ad.C28-sCE2 and characterisation of Ad.C28-sCE2-transduced SWI398 cells by Western blot analysis, esterase activity assay and immunohistochemistry. (A) Schematic structure of the replication-deficient adenovirus Ad.C28-sCE2. The C28-sCE2 expression cassette includes the CMV promoter, an loG $k$ leader sequence for secretion and a C-terminal myc-and His-tag for detection and purification. The adenovirus also contains the gene encoding GFP under the CMV promoter. (B) Western blot analysis of cellular lysates (lanes I and 2) and supernatants (lanes 3 and 4) of SWI398 cells transduced with Ad.C28-sCE2 (lanes I and 3) or AdGFP (lanes 2 and 4) at an MOI of I00. C28-sCE2 was detected using an antibody directed to the myc-tag. (C) CE activity in cellular lysates and supernatants of SWI398 cells transduced with Ad.C28-sCE2 or AdGFP at an $\mathrm{MOI}$ of 100. Cellular lysates or supernatants were incubated with I mM pNpAc and conversion was measured during 10 min. C28-sCE2 showed enzymatic activity and was efficiently secreted by transduced cells, since most of the activity was detected in the supernatant. (D) Binding of C28sCE2 to the EpCAM-expressing cell line Colo205. Colo205 cells or the EpCAM-negative cell line A2780 were incubated with the supernatant of SWI 398 cells transduced with Ad.C28-sCE2 or AdGFP at an MOI of I00. After washing, the cells were stained with anti-myc antibody to show binding of C28-sCE2. Only the EpCAM-expressing Colo205 cells incubated with supernatant of Ad.C28-sCE2-transduced SWI398 cells showed a positive membrane staining, indicating that the fusion protein had bound specifically to the Colo205 cells.

with the supernatant of SW1398 cells transduced with Ad.C28sCE2 or AdGFP. As can be seen in Figure 1D, C28-sCE2 specifically bound to the cellular membranes of EpCAM-expressing cells.

\section{Diffusion of C28-sCE2 in multicellular colon cancer tumour spheroids}

Colo205 spheroids were transduced with Ad.C28-sCE2 and cryosections were made 1,4 and 5 days later. Sections were stained with an anti-myc antibody to localise the C28-sCE2 fusion protein. Figure 2 illustrates that on day 1 after transduction only the outer rim of the spheroid stained slightly positive for C28sCE2. Sections of spheroids harvested at later time points after transduction showed the presence of C28-sCE2 in deeper layers of the spheroid. A higher magnification of the anti-myc staining at day 5 after transduction (Figure 2B) suggests that C28-sCE2 had bound untransduced neighbouring cells since only the cellular membrane of these cells stained positive. Thus, C28sCE2 penetrated into and accumulated in the tumour mass surrounding Ad.C28-sCE2-transduced cells. 
A

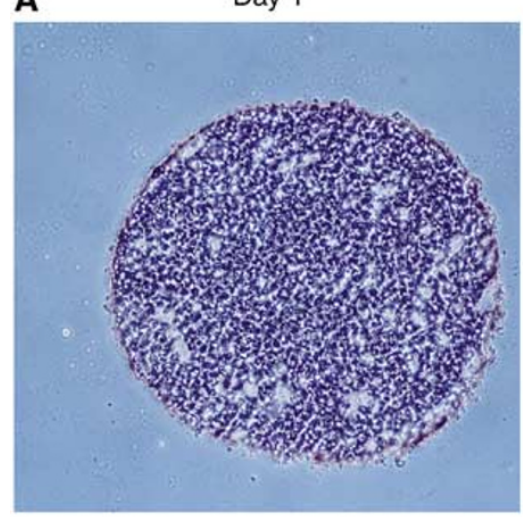

Day 4

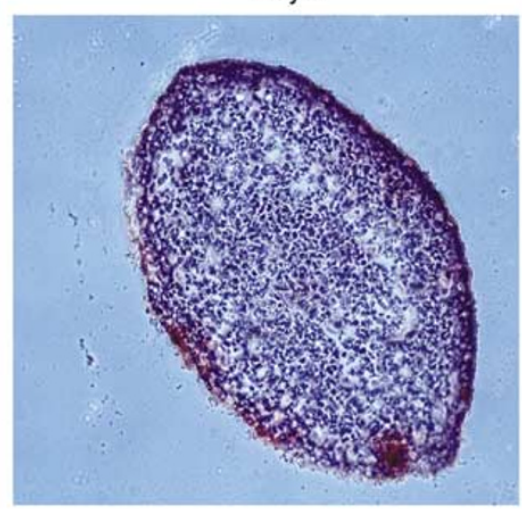

Day 5

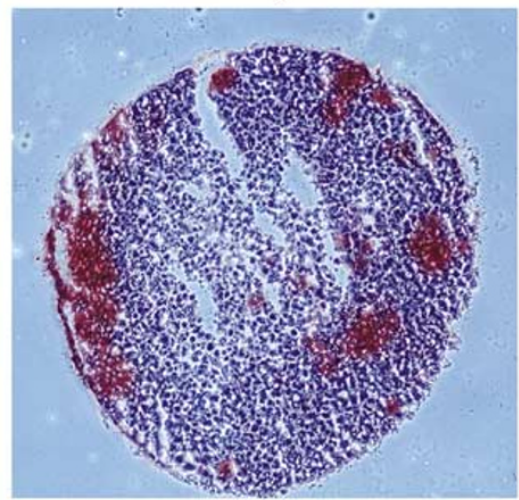

B

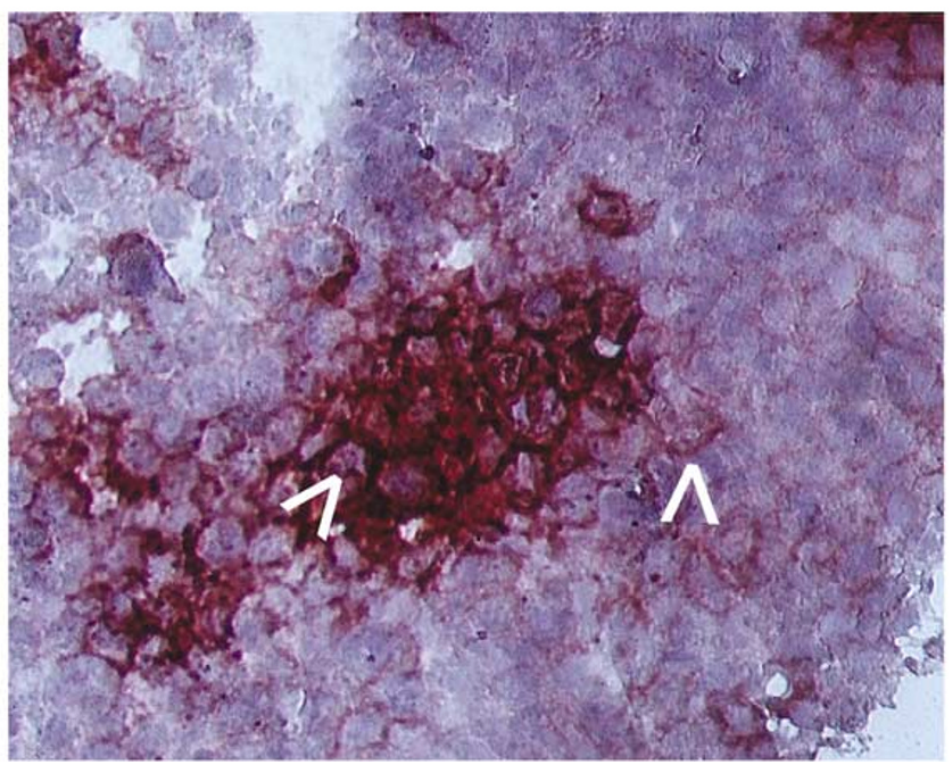

Figure 2 Immunohistochemistry on sections of Ad.C28-sCE2-transduced Colo205 spheroids. Colo205 spheroids were transduced with I $\times 10^{7}$ PFU Ad.C28-sCE2 and harvested at day I, 4 and 5 after transduction. Sections of these spheroids were made and stained for myc to detect C28-sCE2. (A) At day I after Ad.C28-sCE2 transduction, no positive staining can be detected. At days 4 and 5, several spots along the rim of the spheroid are positively stained. (B) A higher magnification of the fusion protein staining at day 5 after transduction is shown. Cells with clear staining of membranes only (arrows) represent untransduced neighbouring cells with bound C28-sCE2.

\section{CPT-11 activation and antiproliferative effects in Ad.C28-sCE2-transduced cells}

Colon cancer spheroids transduced with Ad.C28-sCE2 or AdGFP were subjected to CPT-11 treatment for 7 days. Figure 3 demonstrated the viability of the spheroid as measured by WST1 conversion. Ad.C28-sCE2-transduced Colo205 and SW1398 colon cancer spheroids were sensitised to CPT-11, since CPT-11 treatment to these spheroids was as toxic as treatment with its activate analogue $\mathrm{SN}-38$.

\section{DISCUSSION}

Targeting chemotherapy specifically to tumour cells with GDEPT is expected to increase the antitumour effect, while side effects are decreased. A limitation of adenoviral vector-mediated cancer gene therapy is the poor penetration ability of adenoviral vectors into a solid tumour mass. To improve the efficacy of adenoviral vectormediated GDEPT approaches, secreted prodrug-converting enzymes have been studied (Weyel et al, 2000; Oosterhoff et al, 2003). However, secreted enzymes might leak away from the site of the tumour. Therefore, cell surface-tethered forms of prodrug- converting enzymes, such as $\beta$-glucuronidase or carboxypeptidase G2, were developed to prevent leakage of untargeted enzyme from the tumour, while prodrug activation is retained (Spooner et al, 2000; Heine et al, 2001). Another way to prevent diffusion of the enzyme from the tumour is secretion by transduced tumour cells of a fusion protein consisting of an scFv antibody and a prodrugconverting enzyme, which can subsequently bind to tumour cells (De Graaf et al, 2002; Oosterhoff et al, 2002). We hypothesised that the bystander effect achieved by such a secreted targeted prodrugconverting enzyme might be more pronounced than that achieved by a cell surface-tethered form, as the targeted form can diffuse and bind to neighbouring tumour cells. In this study, we investigated the utility of a replication-deficient adenoviral vector containing the cDNA encoding a secreted, EpCAM-targeted form of human liver CE2, Ad.C28-sCE2, to sensitise colon cancer tumours to CPT-11. We chose to study Ad.C28-sCE2 in a threedimensional in vitro colon cancer spheroid model, because the three-dimensional structure of spheroids resembles in vivo tumours much closer than two-dimensional cell cultures. Furthermore, we wanted to visualise the bystander effect by determining secretion of $\mathrm{C} 28-\mathrm{sCE} 2$ and penetration of the fusion protein through a solid tumour mass, which can only be studied in 

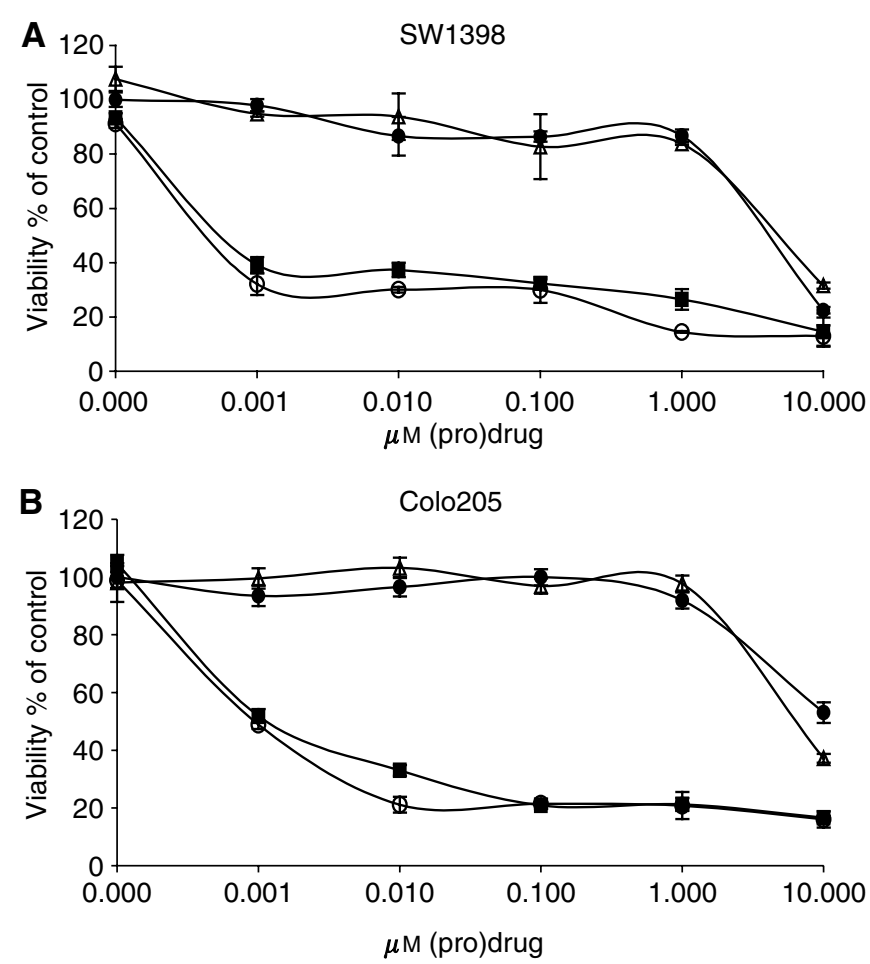

Figure 3 Cytotoxicity assay on SWI398 and Colo205 colon cancer spheroids. SWI 398 (A) and Colo205 (B) spheroids were transduced with I $\times 10^{7}$ PFU AdGFP or Ad.C28-sCE2. At 7 days after infection, spheroids were subjected to a range of CPT-I I concentrations and cultured for a further 7 days. Cell viability of untransduced spheroids treated with CPT-I I (closed black circles) or SN-38 (open black circles), AdGFP-transduced spheroids treated with CPT-I I (open black triangles) and Ad.C28-sCE2transduced spheroids treated with CPT-II (closed black squares) were analysed by WST-I conversion measurement.

a three-dimensional structure. Grill et al demonstrated that transduction of primary glioma spheroids with a replicationdeficient vector resulted in the expression of the transgene in the outer rim of the spheroid only. This showed that spheroids are relevant structures to study lack of adenovirus penetration into solid tumour masses (Grill et al, 2002). In the colon cancer spheroid model used in this study, we were able to detect the C28sCE2 fusion protein bound to untransduced cells several cellular layers away from transduced cells. This suggests that C28-sCE2 is capable of diffusing into a solid tumour mass.

From these results we hypothesised that optimal cytotoxicity from CPT-11 could be expected if the prodrug was administered at least a few days after Ad.C28-sCE2 transduction when C28-sCE2 has spread through the spheroid. Transduction of colon cancer spheroids with Ad.C28-sCE2 and treatment with CPT-11 after 7 days resulted in complete sensitisation of these spheroids to CPT11. The toxicity to these spheroids was comparable to $\mathrm{SN}-38$ treatment, indicating that CPT-11 is effectively converted into the toxic drug.

In order to compare a targeted prodrug-converting enzyme with a secreted prodrug-converting enzyme, it is necessary to perform in vivo experiments. However, the high endogenous plasma esterase activity in mice presents a challenge in using mouse models to evaluate tumour-specific conversion of CPT-11. In mice, more than $50 \%$ of the administered CPT- 11 is converted to $\mathrm{SN}-38$ by plasma esterases (Morton et al, 2000), whereas in human patients less than $5 \%$ of the prodrug is activated (Rivory et al, 1997). Hence, the analysis of CE-mediated activation of CPT-11 in normal mice does not accurately reflect what happens after the administration of the drug to humans. Previously, a strain of plasma esterase-deficient mice was described (Morton et al, 2000), in which CPT-11 metabolism is comparable to that observed in humans. Recently, these mice were crossbred with SCID mice (personal communication with Dr Phil Potter, St Jude Children's Research Hospital, Memphis, USA) and we are currently testing adenoviral vectors expressing EpCAM-targeted sCE2 or untargeted sCE2 in these esterase-deficient SCID mice bearing colon cancer xenografts.

In conclusion, we constructed a replication-deficient adenoviral vector containing a cDNA encoding a secreted, EpCAM-targeted form of human liver CE2 that was capable of converting the prodrug CPT-11 into its activated form, leading to enhanced toxicity of CPT-11 to colon cancer spheroids. Therefore, this adenoviral construct holds promise in GDEPT approaches for the treatment of patients with EpCAM-expressing colon cancer.

\section{ACKNOWLEDGEMENTS}

We thank Jeroen Mastenbroek for his expert technical assistance. Dinja Oosterhoff is supported by an Educational Grant of Aventis. Victor van Beusechem is supported by a research fellowship of the Royal Netherlands Academy of Arts and Sciences (KNAW).

\section{REFERENCES}

Chan S, Gabra H, Hill F, Evan G, Sikora K (1987) A novel tumour marker related to the c-myc oncogene product. Mol Cell Probes 1: 73-82

De Graaf M, Boven E, Oosterhoff D, Van der Meulen-Muileman IH, Huls GA, Gerritsen WR, Pinedo HM (2002) A fully human anti-Ep-CAM scFv beta-glucuronidase fusion protein for selective chemotherapy with a glucuronide prodrug. Br J Cancer 86: 811-819

Grill J, Lamfers ML, van Beusechem VW, Dirven CM, Pherai DS, Kater M, Van der Valk P, Vogels R, Vandertop WP, Pinedo HM, Curiel DT, Gerritsen WR (2002) The organotypic multicellular spheroid is a relevan three-dimensional model to study adenovirus replication and penetration in human tumors in vitro. Mol Ther 6: 609-614

Gupta E, Mick R, Ramirez J, Wang X, Lestingi TM, Vokes EE, Ratain MJ (1997) Pharmacokinetic and pharmacodynamic evaluation of the topoisomerase inhibitor irinotecan in cancer patients. J Clin Oncol 15: $1502-1510$

Haisma HJ, Van Muijen M, Scheffer G, Scheper RJ, Pinedo HM, Boven E (1995) A monoclonal antibody against human beta-glucuronidase for application in antibody-directed enzyme prodrug therapy. Hybridoma 14: $377-382$
He TC, Zhou S, da Costa LT, Yu J, Kinzler KW, Vogelstein B (1998) A simplified system for generating recombinant adenoviruses. Proc Natl Acad Sci USA 95: 2509-2514

Heine D, Muller R, Brusselbach S (2001) Cell surface display of a lysosomal enzyme for extracellular gene-directed enzyme prodrug therapy. Gene Ther 8: $1005-1010$

Humerickhouse R, Lohrbach K, Li L, Bosron WF, Dolan ME (2000) Characterization of CPT-11 hydrolysis by human liver carboxylesterase isoforms hCE-1 and hCE-2. Cancer Res 60: 1189-1192

Kojima A, Hackett NR, Ohwada A, Crystal RG (1998) In vivo human carboxylesterase cDNA gene transfer to activate the prodrug CPT-11 for local treatment of solid tumors. J Clin Invest 101: 1789-1796

Laemmli UK (1970) Cleavage of structural proteins during the assembly of the head of bacteriophage T4. Nature 227: 680-685

Litvinov SV, Bakker HA, Gourevitch MM, Velders MP, Warnaar SO (1994) Evidence for a role of the epithelial glycoprotein 40 (Ep-CAM) in epithelial cell-cell adhesion. Cell Adhes Commun 2: 417-428

Morton CL, Wierdl M, Oliver L, Ma MK, Danks MK, Stewart CF, Eiseman JL, Potter PM (2000) Activation of CPT-11 in mice: identification 
and analysis of a highly effective plasma esterase. Cancer Res 60: $4206-4210$

Oosterhoff D, Pinedo HM, van der Meulen IH, de Graaf M, Sone T, Kruyt FA, van Beusechem VW, Haisma HJ, Gerritsen WR (2002) Secreted and tumour targeted human carboxylesterase for activation of irinotecan. $\mathrm{Br} J$ Cancer 87: 659-664

Oosterhoff D, Witlox MA, van Beusechem VW, Haisma HJ, Schaap GR, Bras J, Kruyt FA, Molenaar B, Boven E, Wuisman PI, Pinedo HM, Gerritsen WR (2003) Gene-directed enzyme prodrug therapy for osteosarcoma: sensitization to CPT-11 in vitro and in vivo by adenoviral delivery of a gene encoding secreted carboxylesterase-2. Mol Cancer Ther 2: $765-771$

Rivory LP, Haaz MC, Canal P, Lokiec F, Armand JP, Robert J (1997) Pharmacokinetic interrelationships of irinotecan (CPT-11) and its three major plasma metabolites in patients enrolled in phase I/II trials. Clin Cancer Res 3: $1261-1266$

Satoh T, Hosokawa M, Atsumi R, Suzuki W, Hakusui H, Nagai E (1994) Metabolic activation of CPT-11, 7-ethyl-10-[4-(1-piperidino)-1-piperidi- no]carbonyloxycamptothecin, a novel antitumor agent, by carboxylesterase. Biol Pharm Bull 17: 662-664

Spooner RA, Martin J, Friedlos F, Marais R, Springer CJ (2000) In suicide gene therapy, the site of subcellular localization of the activating enzyme is more important than the rate at which it activates prodrug. Cancer Gene Ther 7: $1348-1356$

Tsuji T, Kaneda N, Kado K, Yokokura T, Yoshimoto T, Tsuru D (1991) CPT-11 converting enzyme from rat serum: purification and some properties. J Pharmacobiodyn 14: 341-349

van Beusechem VW, van Rijswijk AL, van Es HH, Haisma HJ, Pinedo HM, Gerritsen WR (2000) Recombinant adenovirus vectors with knobless fibers for targeted gene transfer. Gene Ther 7: 1940-1946

Weyel D, Sedlacek HH, Muller R, Brusselbach S (2000) Secreted human beta-glucuronidase: a novel tool for gene-directed enzyme prodrug therapy. Gene Ther 7: 224-231

Wierdl M, Morton CL, Weeks JK, Danks MK, Harris LC, Potter PM (2001) Sensitization of human tumor cells to CPT-11 via adenoviral-mediated delivery of a rabbit liver carboxylesterase. Cancer Res 61: 5078-5082 